\title{
On the spectrum of discrete Schrödinger equation with one-dimensional perturbation 1
}

\author{
V. V. Borzov ${ }^{a}$ E. V. Damaskinsky ${ }^{b}$ \\ ${ }^{a}$ Department of Mathematics, St.Petersburg State University of Telecommunications, \\ Prospekt Bolshevikov 22-1, St.Petersburg, 193232, Russia; borzov.vadim@yandex.ru \\ ${ }^{b}$ Mathematical Department, VI(IT), Russia, 191123, Zacharievskaya 22, St.Petersburg, Russia; \\ evd@pdmi.ras.ru
}

\begin{abstract}
We consider the spectrum of the discrete Schrödinger equation with one-dimensional perturbation. We obtain the explicit form of scattering matrix and find the exact condition of absence of singular part of the spectrum. We calculated also the eigenvalue that appears if this condition is not true. In the last part of our paper we give few remarks on the case of two-dimensional perturbations.
\end{abstract}

\section{Introduction}

'The problem of a change in the spectrum of self-adjoint operator with one-dimensional perturbation in the well-known Friedrichs - Faddeev model [1, [2] on a finite segment $[a, b]$ is well studied. Description of this model and references see in the monograph [3]. Let $v(\lambda, \tau)=\beta u(\lambda) \overline{u(\tau)}$ is the kernel of the integral operator of the perturbation. The function $v(\lambda, \tau)$ satisfies the Hölder condition with index $\alpha_{0}$, and $u(a)=u(b)=0$. It is well-known that in the case of one-dimensional perturbation the absolutely continuous part of the spectrum not changed, and the singular part of the continuous spectrum for arbitrary function $u(\lambda)$ does not only when $\alpha_{0}>\frac{1}{2}$. It is also known

\footnotetext{
${ }^{1}$ This article is extended variant of the manuscript with the same title submitted to the Proceedings of the Days on Diffraction 2016
} 
that if the coupling constant $\beta$ is small, then the discrete spectrum of the perturbed operator from outside the segment $[a, b]$ is missing. However, there is some positive critical value $\beta_{0}$ such that if $|\beta|>\beta_{0}$, then there is one simple eigenvalue $\lambda$ from outside the segment $[a, b]$. In the general case, even for one-dimensional perturbations, provided that $\alpha_{0} \leq \frac{1}{2}$ the singular spectrum can be quite complicated (see theorem 6.7.10 in [3]).

In this paper we consider a special case of one-dimensional perturbations in Friedrichs-Faddeev model on the finite segment $[-2,2]$. Namely the case when the kernel of the integral operator of the perturbation takes the form $u(\lambda)=U_{k}\left(\frac{\lambda}{2}\right) f(\lambda),\left(k \in Z_{+}\right)$where

$$
f(\lambda)=\frac{1}{\pi} \sqrt{1-\frac{\lambda^{2}}{4}} .
$$

and $U_{n}$ are the Chebyshev polynomials of the 2-nd kind. These polynomials satisfy the following recurrence relations

$$
\lambda U_{n}\left(\frac{\lambda}{2}\right)=U_{n+1}\left(\frac{\lambda}{2}\right)+U_{n-1}\left(\frac{\lambda}{2}\right), \quad n \geq 1, \quad U_{0}\left(\frac{\lambda}{2}\right)=1, \quad U_{1}\left(\frac{\lambda}{2}\right)=\lambda .
$$

In our case the function $u(\lambda)$ equals to zero at the ends of the segment and satisfies the Hölder condition with index $\alpha_{0}=\frac{1}{2}$.

Taking into account possible applications of our results it is convenient to consider the model as a discrete Schrödinger operator with a local one-dimensional perturbation. This model is found in the study of some problems of atomic physics (see [6]), and also in the investigation of non-equivalent representations algebra of generalized Chebyshev oscillator (see [7], [8]).

Let us turn to a precise formulation of the problem. We give the definition of the considered Schrödinger operator following $\S 4.1$ of the monograph [4]. Take as a starting point, the Jacobi $\operatorname{matrix} \mathcal{J}_{k}^{(\beta)}=\left\{j_{i, n}^{(\beta, k)}\right\}_{i, n=0}^{\infty}$, where

$$
j_{i, n}^{(\beta, k)}=\left\{\begin{array}{rll}
1, & \text { if } & |i-n|=1, \\
\beta \delta_{n k}, & \text { if } & i=n, \\
0, & \text { if } & |i-n|>1,
\end{array}\right.
$$

$\delta_{n k}$ is the Kronecker delta and $\beta$ is a real number. 
Let $\mathcal{H}_{k}=\mathrm{L}_{2}\left(\mathcal{R} ; \mu_{k}\right)$ be a separable Hilbert space where $\mu_{k}$ is a Borel probability measure, such that

$$
\nu_{n}=\int_{-\infty}^{+\infty} \lambda^{n} \mu_{k}(d \lambda)<\infty, \quad n \geq 0,
$$

and $\nu_{0}=1$. We consider $\mathcal{J}_{k}^{(\beta)}$ as the matrix of linear operator $H_{k}^{(\beta)}$ in $\mathcal{H}_{k}$ defined on the orthonormal basis $\left\{\phi_{n}^{(k)}(\lambda)\right\}_{n=0}^{\infty}$ of the space by relations

$$
\begin{gathered}
H_{k}^{(\beta)} \phi_{n}^{(k)}=\phi_{n+1}^{(k)}+\phi_{n-1}^{(k)}+\beta \delta_{n k} \phi_{n}^{(k)}, \quad n=1,2, . . \\
H_{1}^{(\beta)} \phi_{0}^{(k)}=\beta \delta_{0 k} \phi_{0}^{(k)}+\phi_{1}^{(k)} .
\end{gathered}
$$

The symmetric operator $H_{k}^{(\beta)}$ is defined on the set of finite linear combinations of basis vectors. The set is dense in the space $\mathcal{H}_{k}$. It is known [4] that the deficiency indices of the operator $H_{k}^{(\beta)}$ are equal to $(0,0)$. Hence its closure is a self-adjoint operator with a simple spectrum in $\mathcal{H}_{k}$. We denote this operator by the same symbol $H_{k}^{(\beta)}$. The operator $H_{k}^{(\beta)}$ defined by (2), (3) is considered discrete Schrödinger operator with a local one-dimensional perturbation.

Further, denote by $\mathcal{J}_{0}$ the Jacobi matrix $\mathcal{J}_{0}=\mathcal{J}_{k}^{(0)}$ and by $H_{0}$ the corresponding self-adjoint operator $H_{0}=H_{k}^{(0)}$.

In the following we will assume that $\mathcal{H}_{k}$ is a space of the spectral representation, i.e. the operator $H_{k}^{(\beta)}$ in the space $\mathcal{H}_{k}$ is the operator of multiplication by the variable $\lambda$.

It is known [9] that the operator $H_{0}$ in spectral representation defined on the Hilbert space $\mathcal{H}_{0}=\mathrm{L}_{2}\left(\mathcal{R} ; \mu_{0}\right)$ with

$$
d \mu_{0}(\lambda)=\frac{1}{\pi}\left\{\begin{array}{ccc}
\sqrt{1-\frac{\lambda^{2}}{4}} d \lambda, & \text { if } & |\lambda| \leq 2, \\
0, & \text { if } & |\lambda|>2
\end{array}\right.
$$

The Chebyshev polynomials of the 2-nd kind $\left\{U_{n}\left(\frac{\lambda}{2}\right)\right\}_{n=0}^{\infty}$ form an orthonormal basis in the space $\mathcal{H}_{0}$. "Perturbed" operator $H_{k}^{(\beta)}$ defined on the Hilbert space $\mathcal{H}_{k}$, where the polynomials $\left.\left\{\phi_{n}^{(k)}(\lambda)\right)\right\}_{n=0}^{\infty}$ form an orthonormal basis. These polynomials satisfy the following recurrence relations

$$
\begin{gathered}
\lambda \phi_{n}^{(k)}(\lambda)=\phi_{n+1}^{(k)}(\lambda)+\beta \delta_{n k} \phi_{n}^{(k)}(\lambda)+\phi_{n-1}^{(k)}(\lambda), \quad n \geq 1, \\
\phi_{0}^{(k)}(\lambda)=1, \quad \phi_{1}^{(k)}(\lambda)=\left(\lambda-\beta \delta_{0 k}\right) .
\end{gathered}
$$


Our aim is to study the singular part of the spectrum of self-adjoint "perturbed"

Hamiltonian $H_{k}^{(\beta)},(\beta \in \mathbb{R})$ for arbitrary $k \in Z_{+}$. This operator occurs after adding onedimensional perturbation to the "free" Hamiltonian $H_{0}$ (corresponding to the case $\beta=0$ ). We use a new representation of the resolvent $R_{0}(z)=\left(H_{0}-z\right)^{-1}$ of self-adjoint unperturbed operator $H_{0}$ in $\mathcal{H}_{0}$. By $(.,$.$) we denote the scalar product in the Hilbert space \mathcal{H}_{0}$. Computing the roots of the denominator of the resolvent

$$
D(z)=1+\beta\left(R_{0}(z) U_{k}, U_{k}\right)
$$

on the real axis, we obtain the following results:

1. Exact condition of the absence of singular spectrum on the continuous spectrum (on the segment $[-2,2])$.

2. The critical value $\beta_{0}$ of the coupling constant $\beta$ equals to $\frac{1}{k+1}$.

3. The boundary points \pm 2 of the continuous spectrum are resonances.

4. The explicit dependence $\lambda=\lambda(\beta)$ of the coupling constant $\beta$ the eigenvalue $\lambda$ which is outside the segment $[-2,+2]$.

5. The explicit form of the scattering matrix.

Besides, we build the orthogonality measure $\mu_{k}$ of the polynomials $\phi_{n}^{(k)}(t)$.

\section{The orthogonality measure $d \mu_{k}$}

We consider Jacobi matrix $\mathcal{J}_{k}^{(\beta)}$ and the matrix

$$
B_{k}=\left\{j_{i, m}^{(k)}\right\}_{i, m=1}^{\infty}, \quad j_{i, m}^{(k)}=\beta \delta_{i, k} \delta_{m, k}
$$

for $\beta \in \mathbb{R}$. Denote by $V_{k}$ the one-dimensional self-adjoint operator corresponding to the matrix $B_{k}$ in a Hilbert space $\mathcal{H}_{0}$. We consider the "perturbed" operator $H_{k}^{(\beta)}$, corresponding to the Jacobi matrix $\mathcal{J}_{k}^{(\beta)}$ in $\mathcal{H}_{0}$, as a sum of $H_{0}$ and $V_{k}$ :

$$
H_{k}^{(\beta)}=H_{0}+V_{k}, \quad V_{k}=\beta\left(., U_{k}\right) U_{k}, \quad\left\|U_{k}\right\|=1, \quad \beta=\bar{\beta} .
$$


We will compute the density $\mu_{k}^{\prime}(\lambda)$ of a measure $\mu_{k}$. Let $E_{0}(\lambda)=E_{0}(-\infty, \lambda)$ and $E_{k}^{(\beta)}(\lambda)=$ $E_{k}^{(\beta)}(-\infty, \lambda)$ - generating functions of the spectral measures $E_{0}$ and $E_{k}^{(\beta)}$ of self-adjoint operators $H_{0}$ and $H_{k}^{(\beta)}$. Let's denote by $L_{k}, L_{k}^{(\beta)}-$ linear spans of the sets $\left\{E_{0}(X) U_{k}\right\}$ and $\left\{E_{k}^{(\beta)}(X) U_{k}\right\}$, respectively. Then restrictions $\tilde{H}_{0}, \tilde{H}_{k}^{(\beta)}$, of the operators $H_{0}$ and $H_{k}^{(\beta)}$ to the subspace $L_{0}=L_{k}^{(\beta)}$ of Hilbert space $\mathcal{H}_{0}$ have a simple spectrum. Therefore, they are multiplication operators on $\lambda$ in $\mathrm{L}_{2}\left(\mathbf{R} ; \varrho_{0}^{(k)}\right)$ and $\mathrm{L}_{2}\left(\mathbf{R} ; \varrho_{k}\right)$, where the measure $\varrho_{0}^{(k)}$ (we denote $\varrho_{0}^{(0)}$ by $\left.\varrho_{0}\right)$ and $\varrho_{k}$ are defined by equalities

$$
\varrho_{0}^{(k)}(.)=\left(E_{0}(.) U_{k}, U_{k}\right), \quad \varrho_{k}(.)=\left(E_{k}^{(\beta)}(.) U_{k}, U_{k}\right) .
$$

We introduce also the measure $\varrho_{k, 0}$

$$
\varrho_{k, 0}(.)=\left(E_{k}^{(\beta)}(.) U_{0}, U_{0}\right)
$$

It is known that

$$
\varrho_{0}(.)=\mu_{0}(.), \quad \varrho_{k, 0}(.)=\mu_{k}(.)
$$

Then we have

$$
\begin{aligned}
\varrho_{0}^{(k)^{\prime}}(\lambda) & =\left|U_{k}\left(\frac{\lambda}{2}\right)\right|^{2} \mu_{0}^{\prime}(\lambda), \\
\varrho_{k}{ }^{\prime}(\lambda) & =\left|U_{k}\left(\frac{\lambda}{2}\right)\right|^{2} \mu_{k}^{\prime}(\lambda),
\end{aligned}
$$

where the measure $\mu_{0}$ defined by (44). We consider the resolvent $R_{k}(z)=\left(H_{k}^{(\beta)}-z\right)^{-1}$ of self-adjoint operator $H_{k}^{(\beta)}$ in $\mathcal{H}_{0}$, and denote by $D_{k}(z)$ the following expression

$$
D_{k}(z)=1+\beta\left(R_{0}(z) U_{k}, U_{k}\right) .
$$

From ([3]) we know that $D_{k}(\lambda+i 0) \neq 0$ for almost all $\lambda$ and

$$
\rho_{0}^{(k)^{\prime}}(\lambda)=\left|D_{k}(\lambda+i 0)\right|^{2} \rho_{k}^{\prime}(\lambda) .
$$

Then we get from (8), (9), (10) for almost all $\lambda$

$$
\mu_{k}^{\prime}(\lambda)=\frac{\mu_{0}^{\prime}(\lambda)}{\left|D_{k}(\lambda+i 0)\right|^{2}} .
$$


From (10) and (11) it follows that absolutely continuous parts of the measures $\varrho_{0, a}^{(k)}$ and $\varrho_{k, a}$ are equivalent, i.e. $\sigma_{a}\left(H_{k}^{(\beta)}\right)=\sigma_{0, a}=[-2,2]$.

By the Privalov theorem [5], using the well-known expression for the resolvent of the operator $H_{0}$ via the Cauchy-Stieltjes integral with respect to the spectral measure, we have

$$
\begin{gathered}
\left(R_{0}(\lambda \pm i 0) U_{k}, U_{k}\right)= \pm \pi i \rho_{0}^{(k)^{\prime}}(\lambda)+I^{(k)}(\lambda) \\
I^{(k)}(\lambda)=v \cdot p \cdot \int_{-\infty}^{+\infty}(t-\lambda)^{-1} \rho_{0}^{(k)^{\prime}}(t) d t
\end{gathered}
$$

We get from (8) that

$$
I^{(k)}(\lambda)=v \cdot p \cdot \int_{-2}^{+2}(t-\lambda)^{-1}\left(U_{k}\left(\frac{t}{2}\right)\right)^{2} \mu_{0}^{\prime}(t) d t,
$$

Using recurrence relations (1), for the Chebyshev polynomials one can calculate the integral $I^{(k)}(\lambda) 2$

$$
I^{(k)}(\lambda)=\left\{\begin{array}{rll}
b_{k}(\lambda) U_{k}\left(\frac{\lambda}{2}\right), & \text { if } & |\lambda| \leq 2, \\
(-1)^{k} a^{k+1}(\lambda) U_{k}\left(\frac{\lambda}{2}\right), & \text { if } & |\lambda|>2
\end{array}\right.
$$

where

$$
b_{k}(\lambda)=-\frac{\lambda}{2} U_{k}\left(\frac{\lambda}{2}\right)+U_{k-1}\left(\frac{\lambda}{2}\right)
$$

and

$$
a(\lambda)=-\frac{2}{\lambda} \frac{1}{1+\sqrt{1-\frac{4}{\lambda^{2}}}} .
$$

We have for $|\lambda| \leq 2$

$$
\begin{aligned}
D_{k}(\lambda+i 0) & =1+\beta\left( \pm \pi i \mu_{0}^{\prime}(\lambda) U_{k}^{2}\left(\frac{\lambda}{2}\right)+\widetilde{L}_{k}(\lambda)\right) \\
\widetilde{L}_{k}(\lambda) & =-\frac{\lambda}{2} U_{k}^{2}\left(\frac{\lambda}{2}\right)+U_{k}\left(\frac{\lambda}{2}\right) U_{k-1}\left(\frac{\lambda}{2}\right) .
\end{aligned}
$$

In the case when $|\lambda|>2$ we have

$$
D_{k}(\lambda+i 0)=1+\beta(-1)^{k} a^{k+1}(\lambda) U_{k}\left(\frac{\lambda}{2}\right) .
$$

Using the identity 3

$$
U_{k}^{2}\left(\frac{\lambda}{2}\right)+U_{k-1}^{2}\left(\frac{\lambda}{2}\right)-\lambda U_{k-1}\left(\frac{\lambda}{2}\right) U_{k}\left(\frac{\lambda}{2}\right)=1,
$$

\footnotetext{
${ }^{2}$ See Appendices $1 \& 2$ for the proof
}

${ }^{3}$ See Appendix 3 for proof 
we get

$$
\left|D_{k}(\lambda+i 0)\right|^{2}= \begin{cases}1+\beta(\beta-\lambda) A_{k}+2 \beta B_{k}, & |\lambda| \leq 2 \\ \left(1+(-1)^{k} \beta a^{k+1}(\lambda) A_{k},\right. & |\lambda|>2\end{cases}
$$

where

$$
A_{k}=U_{k}^{2}\left(\frac{\lambda}{2}\right), \quad B_{k}=U_{k}\left(\frac{\lambda}{2}\right) U_{k-1}\left(\frac{\lambda}{2}\right)
$$

Finally, from 4, (111) and (18) it follows that

$$
\mu_{k}^{\prime}(\lambda)=\frac{1}{\pi}\left\{\begin{array}{ccc}
\frac{\sqrt{1-\frac{\lambda^{2}}{4}}}{1+\beta(\beta-\lambda) A_{k}+2 \beta B_{k}}, & \text { if } & |\lambda| \leq 2, \\
0, & \text { if } & |\lambda|>2 .
\end{array}\right.
$$

\section{Scattering Matrix for $\left(H_{0}, H_{k}^{(\beta)}\right)$}

Now we turn to the scattering matrix. According to theorem 6.7.3 ([3]) the scattering matrix $S(\lambda)$ for a pair of self-adjoint operators $H_{0}$ and $H_{k}^{(\beta)}$ is calculated for almost all $\lambda \in \widehat{\sigma}_{0}$ by the following formula

$$
S^{(k)}(\lambda)=I(\lambda)-2 \pi \beta i D_{k}^{-1}(\lambda+i 0)\left\langle., \widetilde{U}_{k}\left(\frac{\lambda}{2}\right)\right\rangle_{h(\lambda)} \widetilde{U}_{k}\left(\frac{\lambda}{2}\right)
$$

Here $\widehat{\sigma}_{0}=[-2,2]$ is the core of the spectrum of the operator $H_{0}$ (the minimal Borel support of the spectral measures $\left.E_{0}\right)$. By $\langle., .\rangle_{h(\lambda)}$ we denote the scalar product in "infinitesimal subspace" $h(\lambda)$ of a direct integral which corresponds to the absolutely continuous part of the operator $H_{0}$. Namely, we denote by $H_{0}^{(a)}$ the restriction of the operator $H_{0}$ on the absolutely continuous subspace $\mathcal{H}_{0}^{(a)}$ of the operator $H_{0}$ and consider the decomposition of the subspace $\mathcal{H}_{0}^{(a)}$ into a direct integral (see [3] for details)

$$
\mathcal{H}_{0}^{(a)} \longrightarrow \int_{\widehat{\sigma}_{0}} \oplus h(\lambda) d \lambda
$$

The element $\widetilde{U}_{k}($.$) in the formula (19) is the representative element of U_{k}$ in the decomposition (20). In our case

$$
\widetilde{U}_{k}\left(\frac{\lambda}{2}\right)=\frac{1}{\sqrt{\pi}} \sqrt[4]{1-\frac{t^{2}}{4}} U_{k}\left(\frac{\lambda}{2}\right) \quad \text { if } \quad|\lambda| \leq 2
$$


Substituting (21) in (19), we get for almost all $\lambda \in[-2,2]$

$$
S^{(k)}=I-\widetilde{I}, \quad|\lambda| \leq 2,
$$

where

$$
\widetilde{I}=\frac{2 \beta \sqrt{\pi} i \sqrt[4]{1-\frac{\lambda^{2}}{4}} U_{k}\left(\frac{\lambda}{2}\right)}{1+\beta\left(i \sqrt{1-\frac{\lambda^{2}}{4}} U_{k}^{2}\left(\frac{\lambda}{2}\right)+\widetilde{L}_{k}(\lambda)\right)}\left\langle., \widetilde{U}_{k}\right\rangle_{h(\lambda)}
$$

and $\widetilde{L}_{k}$ is defined by (16).

\section{Point spectrum of the operator $H_{k}^{(\beta)}$}

According to 6.7.6 [3], if the point $\lambda_{0}$ belongs to the point spectrum $\sigma\left(H_{k}^{(\beta)}\right)$ on the segment $[-2,2]$, then it is a solution of the equation

$$
\left|D_{k}(\lambda+i 0)\right|^{2}=0
$$

According to (18), we rewrite this equation in the form

$$
1+\beta(\beta-\lambda) U_{k}^{2}\left(\frac{\lambda}{2}\right)+2 \beta U_{k}\left(\frac{\lambda}{2}\right) U_{k-1}\left(\frac{\lambda}{2}\right)=0
$$

This is a quadratic equation with respect to the variable $\beta$ :

$$
U_{k}^{2}\left(\frac{\lambda}{2}\right) \beta^{2}-U_{k}\left(\frac{\lambda}{2}\right)\left(\lambda U_{k}\left(\frac{\lambda}{2}\right)-2 U_{k-1}\left(\frac{\lambda}{2}\right)\right) \beta+1=0 .
$$

For any $\lambda$ satisfying the inequality $U_{k}\left(\frac{\lambda}{2}\right) \neq 0$ it is easy to obtain the solution of the quadratic equation

$$
\beta_{ \pm}(\lambda)=\frac{\lambda U_{k}\left(\frac{\lambda}{2}\right)-2 U_{k-1}\left(\frac{\lambda}{2}\right)}{2 U_{k}\left(\frac{\lambda}{2}\right)} \pm \sqrt{\frac{\lambda^{2}}{4}-1} .
$$

Since $\beta \in \mathbb{R}$, from (23) it is clear that if $|\lambda|<2$ solutions of equation (22) does not exist. Hence, we proved the absence of the point spectrum of the operator $H_{k}^{(\beta)}$ in the interval $(-2,2)$.

Consider the boundary points \pm 2 . Those numbers are solutions of the equation (23). More precisely, since

$$
U_{k}(1)=k+1, \quad U_{k}(-1)=(-1)^{k}(k+1),
$$


then

$$
\beta_{ \pm}(2)=\frac{1}{k+1}, \quad \beta_{ \pm}(-2)=-\frac{1}{k+1}
$$

Now we will show that points \pm 2 are not eigenvalues of the operator $H_{k}^{\left( \pm \frac{1}{k+1}\right)}$.

Using recurrence relations (5), (6) it is easy to show that the polynomials $\phi_{n}^{(k)}$ are calculated from the following formulat 4

$$
\begin{gathered}
\phi_{0}^{(k)}=U_{0}, \phi_{1}^{(k)}=U_{1}, \ldots, \phi_{k}^{(k)}=U_{k}, \\
\phi_{k+s}^{(k)}=U_{k+s}-\beta U_{k-1+s}-\beta U_{k-3+s}-\ldots-\beta U_{k+1-s}, \quad 1 \leq s \leq k+1, \\
\phi_{n}^{(k)}=U_{n}-\beta U_{n-1}-\beta U_{n-3}-\ldots-\beta U_{n-(2 k+1)}, \quad n \geq 2 k+2 .
\end{gathered}
$$

From (24) and (25) it follows that

$$
\sum_{n=0}^{\infty}\left|\phi_{n}^{(k)}( \pm 2)\right|^{2} \geq \sum_{n=2 k+2}^{\infty}\left|\phi_{n}^{(k)}( \pm 2)\right|^{2}=\infty
$$

since for $n \geq 2 k+2$ we have

$$
\left|\phi_{n}^{(k)}( \pm 2)\right|^{2}=(k+1)^{2}
$$

Thus the boundary points \pm 2 of the continuous spectrum are not eigenvalues of the operator $H_{k}^{( \pm 1 / k+1)}$.

Now we have to calculate the eigenvalues $\lambda(\beta)$ of the operator $H_{k}^{(\beta)}$ for $|\beta|>\frac{1}{k+1}$, i.e. outside the segment $[-2,+2]$. For this we need to find the solution of equation (22) outside of segment $[-2,+2]$. Taking into account (18), the equation (22) can be written outside of segment $[-2,+2]$ in the following way

$$
1+(-1)^{k} \beta a^{k+1}(\lambda) U_{k}\left(\frac{\lambda}{2}\right)=0 .
$$

As can be seen from (15), the function $a^{k+1}(\lambda) \neq 0$ for $|\lambda|>2$. Then we can find a solution of the equation (26)

$$
\beta=(-1)^{k+1} \frac{-\lambda\left(1+\sqrt{1-\frac{4}{\lambda^{2}}}\right)^{k+1}}{2} \frac{(-\lambda / 2)^{k}}{U_{k}(\lambda / 2)} .
$$

\footnotetext{
${ }^{4}$ See Appendix 4 for the proof
} 
It is easy to see that

$$
\beta(2)=\frac{1}{k+1}, \quad \beta(-2)=-\frac{1}{k+1},
$$

and the function $|\beta(\lambda)|$ is monotonically increasing from $\frac{1}{k+1}$ to $\infty$ if $|\lambda|$ increases monotonically from 2 to $\infty$. Therefore the function $\beta(\lambda)$ has a unique inverse function $\lambda=\lambda(\beta)$ and $|\beta(\lambda)|>\frac{1}{k+1}$ as $|\lambda|>2$. The function $\lambda=\lambda(\beta)$ is the sought eigenvalue.

As shown above when $|\beta| \leq \frac{1}{k+1}$, the operator $H_{k}^{(\beta)}$ has no point spectrum on the segment $[-2,+2]$. Besides, for any $\beta$ such that $|\beta|>\frac{1}{k+1}$ there is some eigenvalue $\lambda(\beta)$ of the operator $H_{k}^{(\beta)}$ on the interval $(2,+\infty)$ (if $\left.\beta>\frac{1}{k+1}\right)$ or on the interval $(-\infty,-2)$ (if $\left.\beta<-\frac{1}{k+1}\right)$.

This means that the boundary points \pm 2 of the continuous spectrum are resonances of operators $H_{k}^{\left( \pm \frac{1}{k+1}\right)}$, respectively.

\section{Two-dimensional perturbation: Case $\vec{k}=(1,2)$}

Now we consider a two-dimensional perturbation of the operator $H_{0}$, which is determined by the Jacobi matrix

$$
B_{1,2}=\left\{j_{i, m}^{(1,2)}\right\}_{i, m=1}^{\infty}, j_{i, m}^{(1,2)}=\beta_{1} \delta_{i 1} \delta_{m 1}+\beta_{2} \delta_{i 2} \delta_{m 2}
$$

for $\beta_{1}, \beta_{2} \in \mathbf{R}$. Denote by $V_{1,2}$ two-dimensional self-adjoint operator, corresponding to the matrix $B_{1,2}$ in the Hilbert space $\mathcal{H}_{0}$. In addition, we consider the Jacobi matrix

$$
\mathcal{J}_{1,2}^{\left(\beta_{1}, \beta_{2}\right)}=\left\{j_{i, m}^{\left(\beta_{1}, \beta_{2}\right)}\right\}_{i, m=1}^{\infty}
$$

where

$$
j_{i, m}^{\left(\beta_{1}, \beta_{2}\right)}=\left\{\begin{array}{rll}
1, & \text { if } & |i-m|=1 \\
\beta_{1} \delta_{i 1} \delta_{m 1}+\beta_{2} \delta_{i 2} \delta_{m 2}, & \text { if } & |i-m| \neq 1 .
\end{array}\right.
$$

Denote by $\Phi_{(1,2)}=\left\{\phi_{n}^{(1,2)}(t)\right\}_{n=0}^{\infty}$ the set of Jacobi polynomials related with the matrix $\mathcal{J}_{1,2}^{\left(\beta_{1}, \beta_{2}\right)}$. 
The polynomials $\Phi_{(1,2)}$ are defined the following recurrence relations

$$
\begin{gathered}
t \phi_{n}^{(1,2)}(t)=\phi_{n+1}^{(1,2)}(t)+a_{n} \phi_{n}^{(1,2)}(t)+b_{n-1} \phi_{n-1}^{(1,2)}(t), \\
\phi_{0}^{(1,2)}=1, n \geq 0, \\
b_{n}=1-\delta_{n, 0}, a_{0}=\beta_{1}, a_{1}=\beta_{2}, a_{n}=0, n \geq 2 .
\end{gathered}
$$

The Jacobi matrix $\mathcal{J}_{1,2}^{\left(\beta_{1}, \beta_{2}\right)}$ corresponds to the operator $H_{1,2}^{\left(\beta_{1}, \beta_{2}\right)}$

$$
\begin{gathered}
H_{1,2}^{\left(\beta_{1}, \beta_{2}\right)}=H_{0}+V_{1,2}, \\
V_{1,2}=\beta_{1}\left(., U_{0}\right) U_{0}+\beta_{2}\left(., U_{1}\right) U_{1} .
\end{gathered}
$$

The polynomials $\Phi_{(1,2)}$ form an orthonormal in $\mathcal{H}_{12}=\mathrm{L}_{2}\left(\mathbf{R} ; d \mu_{1,2}(t)\right)$. Just as it was done previously, we can calculate the measure $\mu_{1,2}$

$$
\begin{gathered}
\mu_{1,2}^{\prime}(t)=\left\{\begin{array}{cc}
\frac{\frac{1}{\pi} \sqrt{1-\frac{t^{2}}{4}}}{Q}, & |t| \leq 2, \\
0, & |t|>2,
\end{array}\right. \\
Q=\beta_{1}^{2}+\left(1-\beta_{1} \beta_{2}\right)^{2}+t\left(2 \beta_{2}-\beta_{1}\left(1+\beta_{1} \beta_{2}+2 \beta_{2}^{2}\right)\right)+t^{2} \beta_{2}\left(\beta_{2}+2 \beta_{1}\right)-t^{3} \beta_{2} .
\end{gathered}
$$

\section{Remarks}

1. Note that if $\beta_{2}=0$, we have $\mu_{1,2}^{\prime}(t)=\mu_{1}^{\prime}(t)$ and if $\beta_{1}=0$, we have $\mu_{1,2}^{\prime}(t)=\mu_{2}^{\prime}(t)$.

2. In contrast to the case of one-dimensional perturbations, for two-dimensional case we can't say that the orthogonality measure $\mu_{1,2}$ for polynomials $\left.\Phi_{(1,2)}(t)=\left\{\phi_{n}^{(1,2)}(t)\right)\right\}_{n=0}^{\infty}$ in the Hilbert space $\mathcal{H}_{12}=\mathrm{L}_{2}\left(\mathbf{R} ; d \mu_{1,2}(t)\right)$ coincides with absolutely continuous part of the measure $\mu_{1,2}$ for some real $\beta_{1} \neq 0, \beta_{2} \neq 0$.

3. However, when $\left|\beta_{1}\right| \leq 1, \quad \beta_{2}=0$ or $\left|\beta_{2}\right| \leq \frac{1}{2}, \quad \beta_{1}=0$, and $\beta_{1}, \beta_{2} \in \mathbf{R}$, the orthogonality measure $\mu_{1,2}$ of the polynomials $\left.\Phi_{(1,2)}(t)=\left\{\phi_{n}^{(1,2)}(t)\right)\right\}_{n=0}^{\infty}$ in $\mathcal{H}_{k}$ coincides with absolutely continuous part of the measures $\mu_{1,2}$.

\section{Acknowledgement}

EVD grateful to RFBR for financial support under the grant 15-01-03148. 


\section{References}

[1] K.O. Friedrichs, Perturbation of Spectra in Hilbert Space, Amer. Math. Soc., Providence, RI, 1965.

[2] L.D. Faddeev, On Friedrichs Model in Theory of Perturbation of continious Spectrum, Proceedings Math. Inst. of Steklov, 73,1964 .

[3] D.R. Yafaev, Mathematical scattering theory: General theory, vol. 105 Transl. Math. Monographs - Amer. Math. Soc., Providence, RI, 1992.

[4] N.I. Akhiezer, The Classical Moment Problem and Some Related Question in Analysis, English translation, Oliver and Boyed, Edinburgh, 1965.

[5] I.I. Privalov, Boundary properties of analytic functions(in Russian),-- Gos. Izdat. Tehn.-Teor. Lit., Moscow - Leningrad, 1950, German transl. I.I. Priwalow, Randeigenschaften analytischer Funktionen, 1956 - VEB Deutscher Verlag, Berlin., 1965.

[6] Yu.N. Demkov, V. N. Ostrovskii, Zero-range potentials and their applications in atomic physics, Plenum Press 1988 (english translation of russian 1975 original).

[7] V.V. Borzov, E.V. Damaskinsky, Composite model for generalized Chebyshev oscillator, Proceedings of the "DAYS ON DIFFRACTION?2010".

[8] V.V. Borzov, E.V. Damaskinsky, Invariance of the generalized oscillator under linear transformation of the related system of orthogonal polynomials, Theor. Math. Phys. (in press).

[9] T.S.Chihara, An Introduction to Orthogonal Polynomials, Gordon and Breach, New York, 1978.

[10] I.S. Gradshteyn, I.M. Ryzhik, Table of Integrals, Series, and Products, Seventh Edition, Academic Press (Translated from Russian by Scripta Technica, Inc.) 2007. 


\section{Appendix 1. The proof of equation (13) when $|\lambda|>2$}

Here we prove the relation

$$
I^{(k)}(\lambda)=\int_{-2}^{+2} \frac{1}{(t-\lambda)}\left(U_{k}\left(\frac{t}{2}\right)\right)^{2} \mu_{0}^{\prime}(t) d t=(-1)^{k} a^{k+1}(\lambda) U_{k}\left(\frac{\lambda}{2}\right), \quad \text { if } \quad|\lambda|>2 .
$$

For this aim we on the first step proved the additional relation

$$
I^{(k)}(\lambda)=\int_{-2}^{+2}\left(\frac{1}{(t-\lambda)}\left(U_{k}\left(\frac{t}{2}\right)\right)^{2} \mu_{0}^{\prime}(t) d t=a \frac{1-a^{2(k+1)}}{1-a^{2}}, \quad \text { if } \quad|\lambda|>2,\right.
$$

where $a=a(\lambda)$ defined by (15) as

$$
a(\lambda)=-\frac{2}{\lambda} \frac{1}{1+\sqrt{1-\frac{4}{\lambda^{2}}}},
$$

from which it follows that

$$
\lambda=-\frac{a^{2}+1}{a} .
$$

On the second step we demonstrate that right hand sides of the relations (27) and (28) are equal, that proves the formula (27).

We will prove formula (28) by induction. First we will check the validity of (28) for $k=0$ and $k=1$. After the change of variable $t=2 \cos x$ in the integral $I^{(0)}(\lambda)$ we obtain

$$
I^{(0)}(\lambda)=\int_{-2}^{+2} \frac{1}{(t-\lambda)} \mu_{0}^{\prime}(t) d t=\frac{2}{\pi} \int_{0}^{\pi} \frac{\sin ^{2} x}{-\lambda+2 \cos x} d x
$$

According to eq. (3.644.4) in [10] at $p=-\lambda, q=2$ and the formula (29), one can show that the last integral is equal to

$$
I^{(0)}(\lambda)=a(\lambda)
$$

Next from the recurrence relations (11) we obtain

$$
U_{k}^{2}=\lambda^{2} U_{k-1}^{2}-2 \lambda U_{k-1} U_{k-2}+U_{k-2}^{2}, \quad k \geq 1
$$

Substituting in (28) the right hand side of equality (32) instead of $U_{k}^{2}$ we have $(k \geq 2)$

$$
I^{(k)}(\lambda)=\int_{-2}^{+2} \frac{t^{2}}{(t-\lambda)} U_{k-1}^{2} \mu_{0}^{\prime}(t) d t-2 \int_{-2}^{+2} \frac{t}{(t-\lambda)} U_{k-1} U_{k-2} \mu_{0}^{\prime}(t) d t+I^{(k-2)}(\lambda) .
$$


Using (17) we replace the product $-t U_{k-1} U_{k-2}$ in this formula by the expression

$$
1-U_{k-1}^{2}-U_{k-2}^{2}
$$

and obtain

$$
I^{(k)}=\int_{-2}^{+2} \frac{t^{2}}{(t-\lambda)} U_{k-1}^{2} \mu_{0}^{\prime}(t) d t+2 a(\lambda)-2 I^{(k-1)}-I^{(k-2)}, \quad k \geq 2
$$

From orthonormality of Chebyshev polynomials with respect to $d \mu_{0}$ and that the function $t U_{k-1}^{2}(t) \mu_{0}^{\prime}(t)$ is odd it follows that the first integral in the right hand side of this equality is equal to

$$
\begin{gathered}
\int_{-2}^{+2} t U_{k-1}^{2} \mu_{0}^{\prime}(t) d t+\lambda \int_{-2}^{+2} U_{k-1}^{2} \mu_{0}^{\prime}(t) d t+\int_{-2}^{+2} \frac{\lambda^{2}}{(t-\lambda)} U_{k-1}^{2} \mu_{0}^{\prime}(t) d t= \\
0+\lambda+\lambda^{2} I^{(k-1)}=\lambda+\lambda^{2} I^{(k-1)}
\end{gathered}
$$

Then we have

$$
I^{(k)}=\lambda+2 a+\left(\lambda^{2}-2\right) I^{(k-1)}-I^{(k-2)}, \quad k \geq 2 .
$$

From (30) we get

$$
\lambda+2 a=\frac{a^{2}-1}{a}, \quad \lambda^{2}-2=\frac{a^{4}+1}{a^{2}} .
$$

Using the relations we can rewrite (33) in the form

$$
I^{(k)}=\frac{a^{2}-1}{a}+\frac{a^{4}+1}{a^{2}} I^{(k-1)}-I^{(k-2)}, \quad k \geq 2 .
$$

Note that when $k=1$ the equality (33) gives

$$
I^{(1)}=\lambda+\lambda^{2} I^{(0)}=\lambda+\lambda^{2} a .
$$

From relations (31) and (35) follows the validity of the formula (28) (and also (27)) for $k=0$ and $k=1$. Now let us prove that by induction the formula (28) is true for any $n \geq 2$. We suppose that (28) is true for $k=n-1$ and $k=n-2$ and prove the validity of this formula for $k=n$. To do this, we substitute into the right side of equality (34) with $k=n$ expression of $I^{(n-1)}$ and $I^{(n-2)}$ obtained from (28) at $k=n-1$ and $k=n-2$, respectively, and check that it coincides with the right hand side of (28) for $k=n$. 
We have

$$
\begin{gathered}
I^{(n)}=\frac{a^{2}-1}{a}+\frac{a^{4}+1}{a^{2}}\left(a+a^{3}+\ldots+a^{2 n-1}\right)-\left(a+a^{3}+\ldots+a^{2 n-3}\right)= \\
-\frac{1}{a}+\left(a^{3}+\ldots+a^{2 n+1}\right)+\left(\frac{1}{a}+a+\ldots+a^{2 n-3}\right)-\left(a^{3}+a^{5}+\ldots+a^{2 n-3}\right)= \\
\left(a+a^{3}+\ldots+a^{2 n+1}\right)=a \frac{1-a^{2(k+1)}}{1-a^{2}}, \quad n \geq 2,
\end{gathered}
$$

i.e. formula (28) are valid for $k=n$.

Now let us prove the validity of formula (27) for $n \geq 2$. Let us suppose that (27) is true for $k=n-1$ and $k=n-2$. Therefore, the right-hand sides of equations (27) and (28) coincide when $k=n-1$ and $k=n-2$, respectively. We will show that then the right part of (27) equal to the right side (28) when $k \geq 2$.

Using the formula (30), we can rewrite recurrence relations (11) in the following form

$$
a U_{n}=-\left(\left(a^{2}+1\right) U_{n-1}+a U_{n-2}\right), \quad n \geq 2 .
$$

This allows us to rewrite (27) as follows

$$
\begin{gathered}
I^{(n)}=(-1)^{n} a^{n+1} U_{n}=(-1)^{n} a^{n}\left(-\left(a^{2}+1\right) U_{n-1}-a U_{n-2}\right)= \\
(-1)^{n-1} a^{n} U_{n-1}\left(a^{2}+1\right)-(-1)^{n-2} a^{n-1} U_{n-2} a^{2}= \\
\left(a^{2}+1\right) I^{(n-1)}-a^{2} I^{(n-2)} .
\end{gathered}
$$

Substituting the expressions $I^{(n-1)}$ and $I^{(n-2)}$ using the formula (28), we obtain

$$
\begin{gathered}
I^{(n)}=\left(a^{2}+1\right)\left(a+a^{3}+\ldots+a^{2 n-1}\right)-a^{2}\left(a+a^{3}+\ldots+a^{2 n-3}\right)= \\
\left(a^{3}+\ldots+a^{2 n+1}\right)+\left(a+a^{3}+\ldots+a^{2 n-1}\right)-\left(a^{3}+a^{5}+\ldots+a^{2 n-1}\right)= \\
\left(a+a^{3}+\ldots+a^{2 n+1}\right)=a \frac{1-a^{2(k+1)}}{1-a^{2}}, \quad n \geq 2 .
\end{gathered}
$$

This expression equals to the right hand side of (28) when $n \geq 2$. Therefore, the formula (27) is true for $k=n$. 


\section{Appendix 2. The proof of equation (13) when $|\lambda| \leq 2$}

It is easy to see that when $|\lambda| \leq 2$ equality (12) can be rewritten as

$$
I^{(k)}(\lambda)=v \cdot p \cdot \int_{-2}^{+2} \frac{1}{(t-\lambda)}\left(U_{k}\left(\frac{t}{2}\right)\right)^{2} \mu_{0}^{\prime}(t) d t=-\frac{\lambda}{2}\left(U_{k}\left(\frac{\lambda}{2}\right)\right)^{2}+L_{k}
$$

where

$$
L_{k}=\int_{-2}^{+2} \frac{1}{(t-\lambda)}\left(U_{k}^{2}\left(\frac{t}{2}\right)-U_{k}^{2}\left(\frac{\lambda}{2}\right)\right) \mu_{0}^{\prime}(t) d t .
$$

We want to prove by induction the following formula

$$
L_{k}(\lambda)=U_{k}\left(\frac{\lambda}{2}\right) U_{k-1}\left(\frac{\lambda}{2}\right), \quad k \geq 0 .
$$

It is obvious that $L_{0}=0$. We compute $L_{1}$ :

$$
L_{1}=\int_{-2}^{+2} \frac{1}{(t-\lambda)}\left(U_{1}^{2}\left(\frac{t}{2}\right)-U_{1}^{2}\left(\frac{\lambda}{2}\right)\right) \mu_{0}^{\prime}(t) d t=\int_{-2}^{+2} t \mu_{0}^{\prime}(t) d t+\lambda \int_{-2}^{+2} \mu_{0}^{\prime}(t) d t=\lambda=U_{1}\left(\frac{\lambda}{2}\right) U_{0}\left(\frac{\lambda}{2}\right),
$$

where the last equality follows from (10).

Using formulas (32) and (17), we rewrite $U_{k}^{2}\left(\frac{t}{2}\right)-U_{k}^{2}\left(\frac{\lambda}{2}\right)$ in the following form

$$
\begin{gathered}
U_{k}^{2}\left(\frac{t}{2}\right)-U_{k}^{2}\left(\frac{\lambda}{2}\right)=\left(t^{2} U_{k}^{2}\left(\frac{t}{2}\right)-\lambda^{2} U_{k}^{2}\left(\frac{\lambda}{2}\right)\right)- \\
\left(2 t\left(-1+U_{k-1}^{2}\left(\frac{t}{2}\right)+U_{k-2}^{2}\left(\frac{t}{2}\right)\right)-2 \lambda U_{k-1}\left(\frac{\lambda}{2}\right) U_{k-2}\left(\frac{\lambda}{2}\right)\right)+ \\
U_{k-2}^{2}\left(\frac{t}{2}\right)-U_{k-2}^{2}\left(\frac{\lambda}{2}\right) .
\end{gathered}
$$

Substituting (39) into (37) we get

$$
L_{k}=\int_{-2}^{+2} \frac{t^{2} U_{k-1}^{2}\left(\frac{t}{2}\right)-\lambda^{2} U_{k-1}^{2}\left(\frac{\lambda}{2}\right)}{t-\lambda} \mu_{0}^{\prime}(t) d t-2 L_{k-1}-L_{k-2}
$$

We write $t^{2} U_{k-1}^{2}\left(\frac{t}{2}\right)-\lambda^{2} U_{k-1}^{2}\left(\frac{\lambda}{2}\right)$ in the form

$$
t^{2} U_{k-1}^{2}\left(\frac{t}{2}\right)-\lambda^{2} U_{k-1}^{2}\left(\frac{\lambda}{2}\right)=\left(t^{2} U_{k-1}^{2}\left(\frac{t}{2}\right)-\lambda^{2} U_{k-1}^{2}\left(\frac{t}{2}\right)\right)+\left(\lambda^{2} U_{k-1}^{2}\left(\frac{t}{2}\right)-\lambda^{2} U_{k-1}^{2}\left(\frac{\lambda}{2}\right)\right) .
$$

Substituting (41) into (40) we have

$$
L_{k}=\lambda+\left(\lambda^{2}-2\right) L_{k-1}-L_{k-2}, \quad k \geq 2 .
$$


Above we show that (38) is hold for $k=0$ and $k=1$. Now we prove it by induction for all $k$. For this end we assume that (38) true for $k=n-1$ and $k=n-2$ and show that it remains true for $k=n$.

From (38) and (42) we have

$$
L_{k}=\lambda+\left(\lambda^{2}-2\right) U_{k-1}\left(\frac{\lambda}{2}\right) U_{k-2}\left(\frac{\lambda}{2}\right)-U_{k-2}\left(\frac{\lambda}{2}\right) U_{k-3}\left(\frac{\lambda}{2}\right)
$$

Then for the proof of formula (38) at $k=n$ it is sufficient to check the validity of the following equality

$$
\lambda+\left(\lambda^{2}-2\right) U_{k-1}\left(\frac{\lambda}{2}\right) U_{k-2}\left(\frac{\lambda}{2}\right)-U_{k-2}\left(\frac{\lambda}{2}\right) U_{k-3}\left(\frac{\lambda}{2}\right)=U_{k}\left(\frac{\lambda}{2}\right) U_{k-1}\left(\frac{\lambda}{2}\right) .
$$

Using recurrent relations (1), we rewrite the previous equality in the form

$$
\lambda U_{k-1}^{2}\left(\frac{\lambda}{2}\right)-U_{k-1}\left(\frac{\lambda}{2}\right) U_{k-2}\left(\frac{\lambda}{2}\right)=\lambda+\left(\lambda^{2}-2\right) U_{k-1}\left(\frac{\lambda}{2}\right) U_{k-2}\left(\frac{\lambda}{2}\right)-U_{k-2}\left(\frac{\lambda}{2}\right) U_{k-3}\left(\frac{\lambda}{2}\right) .
$$

It is easy to see that the equality is equivalent to the following equality

$$
\lambda U_{k-1}^{2}=\lambda+\lambda^{2} U_{k-1} U_{k-2}-\lambda U_{k-2}^{2}
$$

Finally, the last equality is equivalent to the identity (17). Thus (38) as well as (13) are proved.

\section{Appendix 3. The proof of the identity (17)}

We want to prove by induction the following identity

$$
U_{k}^{2}\left(\frac{\lambda}{2}\right)+U_{k-1}^{2}\left(\frac{\lambda}{2}\right)-\lambda U_{k-1}\left(\frac{\lambda}{2}\right) U_{k}\left(\frac{\lambda}{2}\right)=1
$$

It is obvious that the equality holds for $k=0$. We will show that for any $n \geq 1$ this equation is valid when $k=n$, if it is true for $k=n-1$. Using recurrent relation (1), we have

$$
\begin{gathered}
U_{n}^{2}+U_{n-1}^{2}-\lambda U_{n-1} U_{n}= \\
\left(\lambda U_{n-1}-U_{n-2}\right)^{2}+U_{n-1}^{2}-\lambda U_{n-1}\left(\lambda U_{n-1}-U_{n-2}\right)= \\
U_{n-1}^{2}+U_{n-2}^{2}-\lambda U_{n-1} U_{n-2} .
\end{gathered}
$$

The obtained expression is equal to unity according to the inductive assumption. Hence, the formula (17) is proved. 


\section{Appendix 4. The proof of the formula (25)}

Here we construct the polynomials $\left\{\phi_{n}^{(k)}(\lambda)\right\}_{n=0}^{\infty}$ related to the Jacobi matrix $\mathcal{J}_{k}^{(\beta)}$. These polynomials (see (2) $(\sqrt{3})$ ) is determined by following recurrence relations $(n \geq 0)$

$$
\begin{gathered}
\lambda \phi_{n}^{(k)}\left(\frac{\lambda}{2}\right)=\phi_{n+1}^{(k)}\left(\frac{\lambda}{2}\right)+a_{n} \phi_{n}^{(k)}\left(\frac{\lambda}{2}\right)+b_{n-1} \phi_{n-1}^{(k)}\left(\frac{\lambda}{2}\right), \\
\phi_{0}^{(k)}=1, \quad a_{n}=\beta \delta_{n k}, \quad b_{n}=1-\delta_{-1 n} .
\end{gathered}
$$

From recurrent relations (11) and (43), it follows that

$$
\phi_{0}^{(k)}=U_{0}, \quad \phi_{1}^{(k)}\left(\frac{\lambda}{2}\right)=U_{1}\left(\frac{\lambda}{2}\right), \ldots, \phi_{k}^{(k)}\left(\frac{\lambda}{2}\right)=U_{k}\left(\frac{\lambda}{2}\right)
$$

From (44), using the recurrent relations (1) and 43 one obtains by induction that

$$
\begin{gathered}
\phi_{k+1}^{(k)}=U_{k+1}-\beta U_{k}, \\
\phi_{k+2}^{(k)}=U_{k+2}-\beta U_{k+1}-\beta U_{k-1}, \ldots, \\
\phi_{2 k+1}^{(k)}=U_{2 k+1}-\beta U_{2 k}-\beta U_{2 k-2}-\ldots-\beta U_{0} .
\end{gathered}
$$

Next, we have the following relation

$$
\begin{gathered}
\phi_{2 k+2}^{(k)}\left(\frac{\lambda}{2}\right)=\lambda \phi_{2 k+1}^{(k)}\left(\frac{\lambda}{2}\right)-\phi_{2 k}^{(k)}\left(\frac{\lambda}{2}\right)= \\
\lambda\left(U_{2 k+1}-\beta U_{2 k}-\beta U_{2 k-2}-\ldots-\beta U_{0}\right)-\left(U_{2 k}-\beta U_{2 k-1}-\beta U_{2 k-3}-\ldots-\beta U_{1}\right) .
\end{gathered}
$$

Combining terms in pairs, standing on the same locations in the first and second brackets in the right hand side of equality (45), and using recurrence relations (11), we get

$$
\phi_{2 k+2}^{(k)}=U_{2 k+2}-\beta U_{2 k+1}-\beta U_{2 k-1}-\ldots-\beta U_{1} .
$$

Finally, to check the validity of the formula

$$
\phi_{n}^{(k)}=U_{n}-\beta U_{n-1}-\beta U_{n-3}-\ldots-\beta U_{n-(2 k+1)}, \quad n \geq 2 k+3, \quad k \geq 0,
$$

it is enough to check that the polynomials $\phi_{n}^{(k)}$ defined by equations (46) for $n \geq 2 k+3$ satisfy recurrence relations (43) that for $n \geq k$ have the form

$$
\phi_{n}^{(k)}\left(\frac{\lambda}{2}\right)=\lambda \phi_{n-1}^{(k)}\left(\frac{\lambda}{2}\right)-\phi_{n-2}^{(k)}\left(\frac{\lambda}{2}\right)
$$


Indeed, we have for all $n \geq 2 k+3, k \geq 0$

$$
\begin{gathered}
\phi_{n}^{(k)}-\lambda \phi_{n-1}^{(k)}+\phi_{n-2}^{(k)}= \\
U_{n}-\beta U_{n-1}-\beta U_{n-3}-\ldots-\beta U_{n-(2 k+1)}-\lambda\left(U_{n-1}-\beta U_{n-2}-\beta U_{n-4}-\ldots-\beta U_{n-2 k-2}\right)+ \\
\left(U_{n-2}-\beta U_{n-3}-\beta U_{n-5}-\ldots-\beta U_{n-2 k-3}\right)= \\
\left(U_{n}-\lambda U_{n-1}+U_{n-2}\right)-\beta\left(U_{n-1}-\lambda U_{n-2}+U_{n-3}\right)-\ldots \\
\ldots-\beta\left(U_{n-(2 k+1)}-\lambda U_{n-2(k+1)}+U_{n-2 k-3}\right)=0 .
\end{gathered}
$$

Thus, we proved that for all $k \geq 0$, the polynomials $\phi_{n}^{(k)}$ can be calculated by the following formulas

$$
\begin{gathered}
\phi_{0}^{(k)}=U_{0}, \quad \phi_{1}^{(k)}=U_{1}, \ldots, \phi_{k}^{(k)}=U_{k}, \\
\phi_{k+s}^{(k)}=U_{k+s}-\beta U_{k+s-1}-\beta U_{k+s-3}-\ldots-\beta U_{k-s+1}, \quad 1 \leq s \leq k+1, \\
\phi_{n}^{(k)}=U_{n}-\beta U_{n-1}-\beta U_{n-3}-\ldots-\beta U_{n-(2 k+1)}, \quad n \geq 2 k+2 .
\end{gathered}
$$

